\title{
Continuidad y desempeño exportador en la empresa peruana
}

\author{
Oscar Malca Guaylupo \\ omalca@up.edu.pe \\ Universidad del Pacífico. Lima. Perú \\ Jorge Rubio Donet \\ rubio_jl@up.edu.pe \\ Universidad del Pacífico. Lima. Perú
}

\section{Resumen}

El presente artículo estudia la relación entre la continuidad de las empresas en los mercados internacionales y su desempeño exportador. Para ello, el estudio se centra en las empresas exportadoras peruanas durante el periodo 2000 a 2008, etapa en la cual las exportaciones peruanas tuvieron un crecimiento continuo. Se analizan los resultados alcanzados en los mercados internacionales, y a través del modelo LOGIT establece la relación probabilística entre su continuidad en dichos mercados y el resultado exportador medido en ventas de exportación.

\section{Palabras clave:}

Internacionalización, empresa peruana, exportaciones, mercados internacionales, desempeño exportador 


\section{Introducción}

A la fecha, los estudios disponibles sobre la internacionalización de la empresa peruana son escasos. Que el Perú venga registrando altas tasas de crecimiento en sus exportaciones y cuente con un gran potencial de recursos, justifican el desarrollo de líneas de investigación que contribuyan a mejorar este proceso de internacionalización.

Según Raymond, Kim y Shao (2001), los estudios relacionados al comportamiento de las empresas exportadoras se clasifican en tres grupos: los orientados a analizar los factores motivacionales que impulsan a las empresas a exportar; los enfocados en analizar el desarrollo del proceso que siguen las empresas en los mercados internacionales; y los estudios orientados a la identificación y el análisis de los factores determinantes del desempeño exportador. El presente artículo se ubica en los orientados al análisis del proceso que siguen las empresas, y analiza la relación entre la continuidad de dichas empresas en los mercados internacionales y el resultado exportador medido en ventas de exportación. El periodo de análisis corresponde a los años 2000-2008, lapso durante el cual el Perú tuvo un crecimiento sostenido. Concretamente, se busca estudiar la hipótesis de que a mayor continuidad de las empresas en los mercados internacionales, mejor su desempeño exportador.

Son diversos los marcos teóricos que demuestran que la continuidad en los mercados genera una gran experiencia que debe ser capitalizada. En consecuencia, es una de las formas que permitiría a las empresas mejorar tanto su desempeño como avanzar en los diferentes modelos y estrategias de internacionalización.

Dado que la actividad exportadora es un proceso dinámico y que las necesidades de apoyo a las empresas varían de acuerdo con su experiencia (Calderón y Fayos, 2004), el análisis de la continuidad exportadora permitiría al Estado peruano examinar y, eventualmente, diseñar políticas orientadas a mejorar la posición competitiva de la empresa exportadora peruana y, con ello, contribuir a la sostenibilidad de las mismas.

El propósito del presente trabajo es demostrar que la continuidad de las empresas en la actividad exportadora influye positivamente en la probabilidad de éxito exportador a largo plazo de la empresa.

\section{Marco Conceptual}

\section{Importancia de la exportación}

La exportación tiene un rol fundamental en la actividad económica tanto en países desarrollados como en vías de desarrollo, principalmente por los beneficios macroeconómicos y microeconómicos derivados del comercio exterior. Desde la perspectiva macroeconómica, la actividad exportadora contribuye al enriquecimiento de las reservas internacionales de cada país, a la promoción del empleo y a mejorar el nivel de vida de sus habitantes (Nguyen, 2000).

En términos microeconómicos, provee a las firmas de ventajas competitivas que contribuyen a mejorar su posición financiera, e incrementar el uso de su capacidad instalada y de la tecnología. En este sentido, en medio de un entorno globalizado, las compañías se benefician de la exportación debido a un incremento en ventas, 
economías de escala que originan menores costos unitarios y que permiten alcanzar mayor rentabilidad, competitividad y productividad (Nguyen, 2000).

Asimismo, en el proceso de internacionalización de las empresas, la exportación es el principal método de entrada a los mercados extranjeros. De hecho, existen diversos estudios empíricos que apoyan la existencia de una relación positiva entre la comercialización de exportaciones y las preferencias de los gerentes por esta estrategia para alcanzar los objetivos de la empresa (Cavusgil \& Nevin, 1981). De acuerdo con Madsen (1998), el análisis del resultado de la actividad exportadora es uno de los factores claves para la toma de decisiones en el desarrollo del comercio internacional.

\section{Internacionalización de la empresa y el modelo de UPPSALA}

Si bien es cierto que la exportación es el punto de inicio del proceso de internacionalización que siguen las empresas, también lo es que el desarrollo del mismo genera relaciones estables entre las empresas exportadoras y los mercados internacionales, las cuales se desarrollan a través de un largo proceso que exige cada vez mayor compromiso con los mercados internacionales (Welch \& Luostarinen, 1988) (Roots, 1994) (Rialp, 1999).

De acuerdo con Johanson \& Vahlne (1977, 1990 y 2009), quienes desarrollaron el modelo de UPPSALA -también conocido como modelo gradualista-,la internacionalización es un proceso de aprendizaje gradual con un creciente compromiso de recursos orientados al mercado internacional por parte de las empresas. Tal como ellos sustentan, este proceso se da en diversas etapas a través de las cuales la empresa incrementa su compromiso con el mercado internacional basado en un mayor conocimiento del mercado el cual incide en la formulación y gestión de decisiones y actividades orientadas a la internacionalización de la empresa.

El proceso de internacionalización de la empresa se da de forma dinámica, de tal manera que las responsabilidades que adquiere en los mercados internacionales requieren cada vez decisiones más comprometidas, así como actividades continuas que enriquecen el conocimiento de mercado de manera incremental (Johanson \& Vahlne, The Mechanism of Internationalisation, 1990).

Asimismo, las empresas van conociendo y aprovechando las oportunidades que se presenten en los mercados. Esto exige mayor compromiso en el desarrollo de sus relaciones en los mercados que les permitan un aprendizaje basado en la construcción de lazos confiables, lo que contribuiría a mejorar su posicionamiento en los mercados internacionales (Johanson \& Vahlne, 2009).

En resumen, el desarrollo internacional tal como lo propone el modelo de UPSSALA implica un constante aprendizaje, por lo cual la continuidad en los mercados internacionales de la empresa exportadora es fundamental para capitalizar este proceso que le permita mejorar su desempeño exportador. 


\section{Recursos y capacidades que influyen en el desempeño exportador}

Para enfrentar los retos de la internacionalización, las empresas deben utilizar todos los recursos a su alcance que le permitan acceder y tener un buen desempeño en los mercados internacionales. Según Peng (2009), la internacionalización implica la interacción de diferentes teorías, tales como las consideraciones basadas en la industria en la que se desempeña la empresa; el enfoque basado en las instituciones, que analizan las restricciones para poder acceder y desarrollarse en los mercados externos, y el análisis centrado en los recursos y capacidades de la empresa.

De acuerdo con Grant (2004), "la empresa es esencialmente un conjunto de recursos y capacidades, los cuales son los determinantes principales de su estrategia". Por ello, el enfoque basado en los recursos y las capacidades destaca un carácter singular de cada empresa y propone que la clave del éxito no recae en hacer lo mismo que otras empresas, sino en explotar las diferencias (Grant, 2004). Así, los recursos y las capacidades están constituidos por los activos tangibles e intangibles que una empresa posee y utiliza para su internacionalización (Peng, 2009) (Grant, 2004), y constituyen la fuente principal de las ventajas competitivas que dicha organización desarrolla (Prahalad \& Hamel, 1990).

Sin embargo, debido a que las empresas comparten entorno y recursos naturales, y dada la complejidad de los mercados extranjeros, las decisiones de cada compañía exportadora en cuanto a sus estrategias se definen sobre la base de los recursos y las capacidades con los que cuenta, con el objeto de captar y retener clientes, así como de alcanzar las metas establecidas (Bettis \& Prahalad, 1995; Navarro, Acedo, Robson, Ruzo, \& Losada, 2010; Cavusgil \& Zou, 1994).

Acceder y mantenerse en los mercados internacionales requiere de una gran inversión en recursos. De acuerdo al modelo basado en la teoría de capacidades organizativas, son diversas las variables que intervienen en las decisiones de internacionalización que afectan el desempeño de la empresa exportadora, las mismas que han sido largamente estudiadas. Nos referimos al tamaño empresarial (Cavusgil \& Nevin, 1981; Bonaccorsi, 1992; Suárez, Olivares \& Galván, 2002; Arteaga \& Medina, 2006; Kogut \& Zander, 2003), al desempeño de los directivos (Del Río \& Varela, 2006), a la estrategia exportadora de la empresa (Porter, 1985), a la naturaleza táctica del know-how, a la experiencia internacional (Kogut \& Zander, 2003) y a la antigüedad de la empresa (Madrid \& García, 2004; De Lucio, Mínguez, Valero \& Mednik, 2008; Suárez, 2004). Todas estas variables fortalecen la experiencia de cada empresa y constituyen el elemento central para desarrollar conocimiento, gestionarlas en los mercados y superar los obstáculos del desarrollo de sus operaciones (Pla \& León, 2004).

En la medida que las organizaciones ganan mayor experiencia en los mercados externos, aumenta su conocimiento y familiaridad con dicho contexto. Así, el aprendizaje contribuye a su proceso de internacionalización y le permite reducir costos de información e incertidumbre (Cyert \& March, 1992; Navarro, Acedo, Robson, Ruzo \& Losada, 2010). La acumulación de experiencia en los mercados exteriores reduce los costos generados en ellos y genera economías de aprendizaje. Además, incrementa las probabilidades de tener mejores resultados que estimulen la exportación y se evidencia, 
claramente, el efecto positivo de la experiencia en las exportaciones (Alonso \& Donoso, 2000; los autores citan a Huerta y Labeaga, 1992).

Adicionalmente a los costos indicados, debemos tener en cuenta los costos hundidos que ya fueron absorbidos por la empresa cuando decidió ingresar al mercado de exportación. Esto la llevará a que tienda a aprovechar las diferentes oportunidades de los mercados internacionales - que genera una barrera de salida- y, así, opte por mantenerse en los mercados internacionales (Jiménez \& Espinoza, 2007; Roberts \& Tybout, 1997). De igual manera, las compañías sin mayor experiencia tenderán a desaprovechar las oportunidades que se presenten en los mercados de exportación (Katsikeas \& Piercy, 1996).

La experiencia de la empresa es un factor relevante que, por lo general, es medido por el número de años que tiene en el sector (Madrid \& García, 2004). La antigüedad y la experiencia exportadora se ven reflejadas en un mayor compromiso con la exportación, lo que garantiza la continuidad de la empresa en el ámbito internacional (Suárez, 2004). De Lucio Fernández et ál. (2008) indica que los procesos interrumpidos de exportación son parte del aprendizaje de las empresas y, en la medida que tengan mayor número de años exportando, el resultado más probable será la continuidad en la actividad exportadora.

Adicionalmente, la continuidad de las empresas en la actividad exportadora redunda en una mayor experiencia y mejor formación de los directivos, quienes influyen en el dinamismo exportador y, por ende, en la continuidad de la empresa en los mercados internacionales (Del Río \& Varela, 2006). Existen características particulares que diferencian a los directivos de una empresa que se orienta a la exportación, de aquellos que lideran organizaciones enfocadas en el mercado local — por ejemplo, las dedicadas a la enseñanza de idiomas-. Otro punto que establece una relación positiva entre la formación de los directivos y el desempeño exportador de la empresa radica en sus percepciones acerca de las complejidades asociadas con la exportación: si consideran que son oportunidades de las cuales pueden tomar ventaja para favorecer su crecimiento (Madrid \& García, 2004). Estas empresas tienden a ser más productivas y eficientes (Bernard \& Jensen, 1999).

Arteaga, García y Medina (2003) indican que existen dos formas genéricas de experiencia que puede tener la empresa: (i) la exportadora, es decir, los años que lleva dedicándose a la exportación y (ii) el total, que implica los años que han transcurrido desde la fundación del negocio. En la medida que se cuente con estos dos tipos de experiencia, se incrementa la posibilidad de desarrollar filiales de ventas en el extranjero. Alonso y Donoso (2000) demuestran que existe un efecto positivo significativo entre la continuidad de la empresa y la propensión exportadora.

El conocimiento es una función de la experiencia (Johanson \& Vahlne, 1977) y esta, a su vez, es un recurso principal para el aprendizaje organizacional. Por lo tanto, la experiencia en exportación contribuirá en la definición y conformación de capacidades gerenciales específicas requeridas para el desempeño exitoso exportador sostenible (Armario, Rastrollo Horrillo, \& González Robles, 2009).

La evidencia empírica demuestra que un número importante de empresas que ingresan a la actividad exportadora no sobreviven más de un año en dicha gesta. Esto se explica 
por las características propias de cada negocio, que afectan su rendimiento y, por lo tanto, son elementos cruciales para su permanencia y éxito (Madrid \& García, 2004).

De acuerdo a lo desarrollado, podemos ver que es necesario analizar la continuidad de la empresa agroexportadora en los mercados internacionales y su relación con el resultado exportador. El trabajo se hace necesario tanto por el análisis como para contribuir a generar debate sobre la internacionalización de las empresas y aportar evidencias empíricas de países emergentes como el Perú.

\section{Metodología}

El propósito del presente artículo, es analizar la continuidad de la empresa peruana en la actividad exportadora y como esta influye en la probabilidad de éxito en su permanencia en los mercados internacionales.

Para el desarrollo de la presente investigación, se toma como unidad de análisis a las empresas exportadoras del Perú. La identificación de las mismas como de los productos exportados se realizó a través de la base de datos de la SUNAT-Aduanas. Para la clasificación de las exportaciones por sectores se tomó como referencia la desarrollada por la base de datos ADEX - DATATRADE.

En función a la data de cada sector se trabajó con el 100\% de las empresas que registraron exportaciones durante el periodo 2000-2008 (Tabla 1).

Tabla 1: Sectores exportadores

\begin{tabular}{|ll|}
\hline 1 & AGROEXPORTADORAS \\
2 & MADERA \\
3 & METAL-MECÁNICO \\
4 & PRENDAS DE VESTIR \\
5 & TEXTIL \\
6 & VARIOS \\
7 & MINERÍA NO METÁLICA \\
8 & MINERÍA TRADICIONAL \\
& PESCA TRADICIONAL Y NO \\
9 & TRADICIONAL \\
& PETRÓLEO Y GAS \\
10 & NATURAL \\
11 & QUÍMICO \\
& SIDERÚRGICO Y \\
12 & METALÚRGICO \\
\hline
\end{tabular}

El sector agroexportador contempla las empresas de exportación agropecuarias, agroindustriales y las de exportación tradicional. Asimismo, en el sector pesquero se juntaron tanto a las empresas de exportación tradicional como las no tradicionales. La data fue utilizada para realizar el análisis descriptivo de cada sector y el desarrollo del modelo LOGIT. 


\section{Procedimiento del modelo LOGIT}

Para la estimación de los coeficientes del modelo se recurre al método de máxima verosimilitud con el propósito de maximizar las concordancias entre los valores observados de $Y$ con sus correspondientes valores estimados. El proceso de estimación se construye a partir de la siguiente función:

$$
h\left(y_{i}, P_{i}\right)=P_{i}^{y_{i}}\left(1-P_{i}\right)^{-y_{i}} \text {, si } y_{i}=0,1
$$

Para una muestra de tamaño "n", la función de verosimilitud es:

$$
L(\mathbf{b})=f\left(y_{i}, P_{i}\right)=\prod_{i=1}^{n} P_{i}^{y_{i}}\left(1-P_{i}\right)^{1-y_{i}}
$$

siendo su transformación logarítmica:

$$
L L(\mathbf{b})=\sum_{i=1}^{n} y_{i} \operatorname{Ln}\left(P_{i}\right)+\sum_{i=1}^{n}\left(1-y_{i}\right) \operatorname{Ln}\left(1-P_{i}\right)
$$

La primera derivada con respecto a b, (llamada función score) es:

$$
U(\mathbf{b})=\frac{\partial[L L(\mathbf{b})]}{\partial \mathbf{b}}=\mathbf{X}^{\prime}(\mathbf{Y}-\mathbf{P})
$$

donde $\mathrm{P}$ es el vector que contiene las probabilidades estimadas de cada individuo que interviene en el estudio; es decir,

$$
\mathbf{P}=\left(\begin{array}{c}
P_{1} \\
\vdots \\
P_{n}
\end{array}\right)
$$

La segunda derivada con respecto a b, llamada matriz de información o hessiana, es:

$$
H(\mathbf{b})=\frac{\partial^{2}[L L(\mathbf{b})]}{\partial \mathbf{b} \partial \mathbf{b}^{\prime}}=-\mathbf{X}^{\prime} \mathbf{W} \mathbf{X}
$$

donde $\mathrm{W}$ es la siguiente matriz diagonal:

$$
\mathbf{W}=\left(\begin{array}{cccc}
P_{1}\left(1-P_{1}\right) & 0 & \cdots & 0 \\
0 & P_{2}\left(1-P_{2}\right) & \cdots & 0 \\
\vdots & \vdots & \ddots & \vdots \\
0 & 0 & \cdots & P_{n}\left(1-P_{n}\right)
\end{array}\right)
$$

Los valores de $\hat{P}_{i}$ se obtienen con el modelo estimado: 


$$
\hat{P}_{i}=\hat{E}\left[Y=1 / \boldsymbol{x}_{i}\right]=\frac{1}{1+e^{-\left(b_{0}+b_{1} x_{1 i}+\cdots+b_{k} x_{k i}\right)}}=\frac{1}{1+e^{-x^{\prime} \mathbf{b}}}
$$

Para obtener el vector de estimadores b se aplica el método iterativo de Newton Raphson mediante la siguiente fórmula recursiva:

$$
\mathbf{b}_{t}=\mathbf{b}_{t-1}+\left(\mathbf{X}^{\prime} \mathbf{W}_{t-1} \mathbf{X}\right)^{-1} \cdot \mathbf{X}^{\prime}\left(\mathbf{Y}-\mathbf{P}_{t-1}\right)
$$

Este proceso se repite hasta que $\left\|\mathbf{b}_{t}-\mathbf{b}_{t-1}\right\| \approx 0$. Algunos programas consideran también la convergencia a cero de la función score.

Para realizar las pruebas estadísticas de significación se utiliza como estadístico de prueba:

$$
W_{i}=\frac{b_{j}}{S_{b_{j}}}
$$

Bajo la hipótesis nula de que el valor del coeficiente individual es cero, estos estadísticos tendrán una distribución aproximadamente normal estándar.

Del mismo modo, los residuos del modelo definidos como:

$$
e_{i}=\frac{y_{i}-\hat{P}_{i}}{\sqrt{\hat{P}_{i}\left(1-\hat{P}_{i}\right)}}
$$

Tendrán una media cero y una variancia uno, si el modelo es correcto. A partir de estos residuales se tiene que el estadístico $\sum e_{i}^{2}$ permite realizar pruebas de significación sobre la bondad del ajuste del modelo.

\section{Modelo: Análisis a través de un modelo LOGIT}

De acuerdo con el análisis de Barreiro Fernández José et al. (2004), son diversas las investigaciones que han utilizado modelos probabilísticos LOGIT para analizar los diversos impactos tanto de las estrategias de marketing, como de las diversas variables que afectan al resultado exportador. Tomando como base estos estudios, en este trabajo se aplica un modelo LOGIT para analizar la influencia de la experiencia exportadora medida en número de años sobre el rendimiento exportador de la empresa.

Para alcanzar el objetivo propuesto, se ha desarrollado el modelo logístico binario, que establece como relaciones principales la incidencia de la continuidad de la empresa en la probabilidad de éxito de su permanencia en la actividad exportadora. Puesto que la probabilidad como indicador de riesgo es un número que puede estar comprendido entre 0 y 1 -donde 0 expresa que es un hecho imposible y 1 expresa la total certeza de la ocurrencia del mismo-, los valores estimados que se obtiene a través de diferentes 
muestras generan distribuciones simétricas o asimétricas, según el valor verdadero del riesgo real. Si la probabilidad o riesgo es 0.5 , o cercana a este valor, la distribución será aproximadamente simétrica; en cambio, si la probabilidad verdadera a estimar tiene un valor próximo a cero o muy cercano a 1 (valores extremos), la distribución será asimétrica (Toma, Rubio, 2009). Dependiendo de ello, los modelos o enlaces usados para estimar dicho valor de riesgo son llamados enlaces simétricos o enlaces asimétricos.

Dentro de este proceso es conveniente tener presente que pueden darse algunas distorsiones en la medición de los riesgos, (Bazán \& Millones, 2008). Los enlaces asimétricos pueden ser determinantes en los resultados de la medición de los riesgos, sobre todo si la probabilidad de éxito $(\mathrm{Pi})$ es un valor cercano a cero o a uno. Para fines del presente trabajo, consideramos que dicha posibilidad no es alta; es decir, partimos de un escenario donde la probabilidad de que una empresa tenga éxito en los siguientes años, considerando que tiene una buena historia exportadora, es un valor que no está muy alejado de 0.5 . En consecuencia, el enlace logístico que proponemos no cae dentro de los problemas de estimación con enlaces asimétricos.

Como lo expresa Pérez \& Fernández (2005), la regresión logística puede ser utilizada para construir un modelo que permita estimar la probabilidad de que una empresa específica tenga permanencia en el mercado. Por lo tanto, se propone estimar dicha probabilidad con el siguiente modelo logístico binario:

$$
P_{i}=E[Y=1 / X]=\frac{1}{1+e^{-\beta_{0}-\beta_{1} X}}=\frac{1}{1+e^{-Z_{i}}}
$$

donde $\mathrm{Pi}$ representa la probabilidad de que la empresa se mantenga dentro del mercado, $X$ representa el factor que mide la permanencia de las empresa, $Y=1$ significa que la empresa se mantiene dentro del mercado, y $Z_{i}=\beta_{1}+\beta_{2} X$.

Por otro lado, la ecuación anterior puede ser presentada como:

$$
\frac{P_{i}}{1-P_{i}}=e^{z_{i}}=e^{\beta_{0}+\beta_{1} X}
$$

la cual representa la razón de probabilidades (odds rate) a favor de que la empresa se mantenga vigente en el mercado. Es decir, es la razón entre la probabilidad de que la empresa se mantenga dentro del mercado y la probabilidad de que la empresa no permanezca dentro del mercado.

A partir de este modelo estudiamos la influencia de ciertos factores Xi en la permanencia de las empresas en el mercado mediante el siguiente modelo logístico:

$$
\operatorname{Ln}\left[\frac{P_{i}}{1-P_{i}}\right]=Z_{i}=\beta_{0}+\beta_{1} X
$$

donde Pi representa una probabilidad, con un valor comprendido entre 0 y 1. 
Tal como lo indica Gujarati (1999), el valor de Zi varía entre $-\infty$ y $+^{\infty}$, siendo 0 solamente cuando $\mathrm{Pi}=0.5$, pues en este caso se tendrá que la razón es igual a 1 y su logaritmo será cero, y será un indicativo de que la probabilidad de que una empresa se mantenga en el mercado es equivalente a la probabilidad de que la empresa no permanezca en el mercado. En consecuencia, un valor positivo de $\mathrm{Zi}$ indicará que $\mathrm{Pi}>0.5 \mathrm{y}$, por tanto la probabilidad de permanencia de la empresa en el mercado es mayor que la probabilidad de no permanencia en él; mientras que un valor negativo de $\mathrm{Zi}$ indicará que $\mathrm{Pi}<0.5 \mathrm{y}$, por tanto, la probabilidad de permanencia de la empresa en el mercado es menor que la probabilidad de no permanencia en el mercado.

Por otro lado, considerando que $Z i$ es lineal en la variable $X$, el valor de $\beta 1$ mide el cambio en $\mathrm{Zi}$ ocasionado por un cambio unitario en el valor del factor $\mathrm{X} y$, por tanto, un valor positivo de $\beta 1$ indicará que el factor $X$ influye positivamente en la permanencia de la empresa en el mercado, mientras que un valor negativo de $\beta 1$ indicará que el factor $X$ influye negativamente en la permanencia de la empresa en el mercado.

Lo mencionado en los dos párrafos anteriores resulta de especial importancia ya que a partir de ello es posible estimar la cantidad mínima de años en que una empresa debe permanecer en el mercado, con el propósito de que la probabilidad de su permanencia sea superior a su probabilidad de no permanencia. De la ecuación anterior se tiene que la probabilidad de que una empresa sea mayor que 0.5 , y que, por tanto, supere a su probabilidad de no permanencia se presentará cuando:

$$
\operatorname{Ln}\left[\frac{P_{i}}{1-P_{i}}\right]=Z_{i}=\beta_{0}+\beta_{1} X>0
$$

De donde se deduce que una empresa tendrá una probabilidad de permanencia en el mercado mayor que la probabilidad de no permanencia cuando:

$$
X>-\frac{\beta_{0}}{\beta_{1}}
$$

Dentro de este marco teórico, nuestra hipótesis de trabajo es que la permanencia de las empresas en la actividad exportadora constituye un factor que explica en gran medida la posibilidad de éxito de la empresa en su actividad exportadora futura. En este sentido, las variables que se proponen más adelante han sido elaboradas con el propósito de cuantificar la permanencia de las empresas en la actividad exportadora en el periodo 2000-2008.

\section{Data y formulación de variables}

Para realizar el proceso de estimación se realizaron los siguientes pasos:

- Se trabajó con la data de las empresas exportadoras por cada uno los sectores, a excepción del sector denominado -'Varios', debido a que este agrupa distintos rubros de negocios que dificulta la comparación de resultados.

- El periodo de análisis fue 2000-2008, basado en el crecimiento continuo que registró el sector, y la información se obtuvo de la base de datos de la Aduana del Perú. 
- Se depuró la data en base a los montos totales exportados, tomándose en cuenta solo a las empresas cuyos montos superen los US $\$ 1,000.00$ (Se considera que un monto anual de exportación menor a esta cifra corresponde a muestras enviadas al exterior, previas al inicio de una actividad exportadora). Se buscó disminuir la variabilidad de la data, dado que muchos montos de exportación corresponden a las muestras enviadas al exterior.

- Para efectos del análisis, las empresas exportadoras se clasificaron en dos grupos. En el primero (empresas pequeñas) figuran las empresas cuyo monto promedio anual exportado es menor que US $\$ 50,000.00$; en el segundo grupo (empresas grandes) figuran aquellas empresas cuyo monto promedio anual exportado es mayor o igual que US\$ 50,000.00.

Para construir el modelo logístico de pronóstico de riesgo se considera al año 2008 como el objetivo de estimación de éxito; es decir, el modelo debe estimar la probabilidad de permanencia de una empresa en el año 2008, tomando como base la información previa de las exportaciones de los años anteriores. De la información disponible correspondiente a la serie de tiempo se identificaron dos problemas por resolver: la presencia de valores atípicos propios de las diferencias en los montos de las exportaciones anuales de las empresas; y la autocorrelación propia de las series de tiempo que generan problemas de multicolinealidad.

Para resolver el primer problema se generó la variable $\mathrm{Y}$ sobre la base de las exportaciones en el año 2008, según el siguiente criterio:

$Y=1$ si la empresa exportó en el año 2008

Y= 0 si la empresa no exportó en el año 2008

Para resolver el segundo problema se resumió la información de cada empresa en un solo indicador que refleje su comportamiento exportador durante el periodo 2000-2008.

Puesto que el propósito de la investigación es comprobar que la continuidad en la actividad exportadora es un factor importante en el éxito futuro de una empresa, la variable explicativa $X$ fue concebida de modo tal que mida dicho comportamiento $y$, en este sentido, se descartó el monto anual de las exportaciones como una variable explicativa del éxito en la permanencia de las empresas en la actividad exportadora, ya que, por un lado, el valor del monto exportado no mide la continuidad de una empresa en su actividad exportadora y, por otro, puede estar sujeta a la presencia de valores extremos $o$ atípicos que distorsionen significativamente la estimación de un modelo. Para lograr esto se cambió la serie de datos de montos exportados por otra en donde el valor de la serie es:

$$
\begin{aligned}
& \mathrm{Et}=1 \text { si la empresa exportó en el año "t" } \\
& \mathrm{Et}=0 \text { si la empresa no exportó en el año "t" }
\end{aligned}
$$

Considerando esta nueva serie de datos en donde la presencia o ausencia en la actividad exportadora es expresada por un valor 0 o un valor 1 , en el presente estudio se propone utilizar tres variables explicativas alternativas. Cada una de ellas tiene como propósito cuantificar de alguna manera la continuidad en la labor exportadora de las 
empresas. Así, se busca que en cada una de las variables propuestas se tenga que valores pequeños indican poca continuidad, y valores altos sean claros indicadores de que la empresa ha mostrado una mayor permanencia en la actividad exportadora en el periodo 2000-2008. Las variables explicativas que se proponen son:

\section{Variable $\mathrm{Na}$}

Número de años que exportó la empresa en el periodo 2000-2008.

Variable Racha (ver http://www.bioestadistica.uma.es/libro/node150.htm)

Se genera combinando los conceptos establecidos en la prueba de hipótesis de signos y la prueba de rachas. En la primera se determina la diferencia entre dos datos y se asigna un valor $+1,0,0-1$ de acuerdo al signo de la diferencia. Para el estudio se propone algo similar, pero tomando las diferencias con un retardo de un año. Es decir, para calificar la actividad exportadora de una empresa en cada periodo se le otorga un puntaje que tiene como propósito premiar la continuidad en la actividad exportadora, y castigar el abandono de la misma. Tomando como información la exportación realizada en un año (Et) y la exportación realizada en el año anterior (Et-1), el valor del puntaje se asignará según los siguientes criterios:

Puntaje $=0$

Si la empresa no exportó en ambos años. Si Et-1=0 y Et=0

La empresa muestra señales de inactividad exportadora.

Puntaje $=+1$

Si la empresa inicia su exportación, o si continúa exportando. Si Et-1=0 y Et

$>0$, o si Et-1 $>0$ y Et $>0$.

La empresa muestra una presencia en la actividad exportadora.

Puntaje $=-1$

Si la empresa deja de exportar. Si Et-1>0 y Et=0

La empresa abandona la actividad exportadora,

La asignación de los puntajes tenía como objetivo premiar la participación de una empresa en la actividad exportadora con un puntaje +1 , y castigar la actividad de la empresa con un puntaje -1 , en el caso que deje de exportar.

A continuación, se hace uso de los métodos señalados en la prueba de hipótesis de rachas, en donde se evalúan las secuencias en las actividades a fin de comprobar la aleatoriedad o la ocurrencia sistemática de una actividad. Tomando como base los puntajes otorgados a cada periodo, el valor de la variable "Racha" se obtiene sumando los puntajes otorgados a una empresa entre los años 2001 y 2008. Una empresa que se muestra activa en su labor exportadora recibirá puntajes de +1 , en los diferentes periodos y, por tanto, el valor de la suma de sus puntajes será mayor. Es decir, un mayor valor de la variable será indicativo de que la empresa mantuvo una fuerte actividad 
exportadora, mientras que un valor pequeño de la variable será indicativo de que la empresa tuvo poca o ninguna actividad exportadora en el periodo analizado.

\section{Variable CON}

Se genera contando el número de años continuos de actividad exportadora antes del 2008. Una empresa que se muestra activa en su labor exportadora recibirá puntajes elevados, mientras que aquella que no tiene mucho tiempo en la actividad exportadora recibirá un puntaje bajo.

Puesto que las tres variables propuestas tienen como objetivo medir la continuidad de una empresa en su actividad exportadora, se observó una fuerte correlación entre ellas, no siendo posible incluirlas simultáneamente en un mismo modelo.

\section{Resultados}

En esta sección se presentan de manera descriptiva los datos proporcionados por la base de datos ADEX. En la tabla 2 se puede observar las tasas de crecimiento de todos los sectores exportadores, según información proporcionada por ADEX. Los datos del sector agroexportador están conformados por los componentes tradicional, agropecuario y agroindustria. De la misma manera comprende el sector pesca, que incluye lo tradicional y lo no tradicional. El promedio de crecimiento de los sectores textil, y petróleo y gas Natural está entre un rango de $9.88 \%$ y $33.84 \%$, respectivamente. Esto ilustra que entre los años 2000 y 2008 , todos los sectores entraron en una etapa de bonanza.

Tabla 2: Crecimiento de las exportaciones (por sector)

\begin{tabular}{|c|c|c|c|c|c|c|c|c|c|}
\hline Sector & $\begin{array}{c}2000- \\
2001\end{array}$ & $\begin{array}{c}2001- \\
2002 \\
\end{array}$ & $\begin{array}{c}2002- \\
2003 \\
\end{array}$ & $\begin{array}{c}2003- \\
2004\end{array}$ & $\begin{array}{c}2004- \\
2005 \\
\end{array}$ & $\begin{array}{c}2005- \\
2006\end{array}$ & $\begin{array}{c}2006- \\
2007\end{array}$ & $\begin{array}{c}2007- \\
2008\end{array}$ & Promedio \\
\hline AGROEXPORTADORAS & $0.28 \%$ & $18.88 \%$ & $10.56 \%$ & $32.50 \%$ & $19.22 \%$ & $31.32 \%$ & $12.19 \%$ & $29.60 \%$ & $19.32 \%$ \\
\hline MADERA & $10.61 \%$ & $31.12 \%$ & $-4.58 \%$ & $25.58 \%$ & $23.73 \%$ & $26.40 \%$ & $0.03 \%$ & $2.97 \%$ & $14.48 \%$ \\
\hline METAL - MECÁNICO & $70.79 \%$ & $-31.04 \%$ & $-9.76 \%$ & $35.17 \%$ & $42.34 \%$ & $-15.12 \%$ & $35.87 \%$ & $47.21 \%$ & $21.93 \%$ \\
\hline PRENDAS DE VESTIR & $0.41 \%$ & $4.81 \%$ & $23.08 \%$ & $35.24 \%$ & $19.85 \%$ & $12.12 \%$ & $18.34 \%$ & $15.80 \%$ & $16.21 \%$ \\
\hline TEXTIL & $-19.21 \%$ & $-7.00 \%$ & $16.50 \%$ & $22.76 \%$ & $4.54 \%$ & $23.59 \%$ & $23.39 \%$ & $14.48 \%$ & $9.88 \%$ \\
\hline VARIOS & $17.20 \%$ & $9.50 \%$ & $13.33 \%$ & $27.67 \%$ & $19.60 \%$ & $17.41 \%$ & $13.64 \%$ & $13.78 \%$ & $16.52 \%$ \\
\hline MINERIA NO METAIICA & $24.01 \%$ & $17.84 \%$ & $7.94 \%$ & $27.81 \%$ & $25.78 \%$ & $12.34 \%$ & $24.20 \%$ & $4.91 \%$ & $18.10 \%$ \\
\hline MINERIAA TRADICIONAI & $-0.11 \%$ & $18.79 \%$ & $23.16 \%$ & $44.37 \%$ & $44.94 \%$ & $48.36 \%$ & $19.76 \%$ & $2.67 \%$ & $25.24 \%$ \\
\hline $\begin{array}{l}\text { PESCA TRADICIONAL YNO } \\
\text { TRAD. }\end{array}$ & $-0.61 \%$ & $-5.89 \%$ & $-3.33 \%$ & $34.48 \%$ & $17.81 \%$ & $4.82 \%$ & $14.68 \%$ & $20.67 \%$ & $10.33 \%$ \\
\hline PETRÓLEO Y GAS NATURAL & $4.74 \%$ & $13.36 \%$ & $39.01 \%$ & $-7.15 \%$ & $157.27 \%$ & $18.08 \%$ & $28.18 \%$ & $17.23 \%$ & $33.84 \%$ \\
\hline QUIM & $16.29 \%$ & $3.90 \%$ & $23.38 \%$ & $52.38 \%$ & $11.29 \%$ & $11.02 \%$ & $35.14 \%$ & $28.11 \%$ & $22.69 \%$ \\
\hline $\begin{array}{l}\text { SIDERURGICO Y } \\
\text { METALÚRGICO }\end{array}$ & $-11.94 \%$ & $-14.39 \%$ & $18.84 \%$ & $55.55 \%$ & $28.50 \%$ & $84.40 \%$ & $12.82 \%$ & $1.36 \%$ & $21.89 \%$ \\
\hline
\end{tabular}

Fuente: Aduanas - Perí. Elaboración propia.

La tabla 3 indica la cantidad de empresas que llegan a ocupar el $50 \%$ del total de exportaciones por cada sector. Para obtener este resultado se siguieron los siguientes pasos:

a) Se ordenó la lista de empresas con sus respectivos montos exportados, de mayor a menor, para cada periodo de la serie por sector.

b) Se halló la participación de cada empresa dentro de cada periodo por sector. 
c) Se halló la participación acumulada con el objetivo de identificar la cantidad de empresas necesarias para alcanzar el $50 \%$ de participación en cada periodo para cada sector.

Como resultado, la tabla 3 muestra las cantidades de empresas necesarias para alcanzar el $50 \%$ de las exportaciones en cada periodo y sector. Asimismo, se aprecia que las cantidades de empresas que ocupan el $50 \%$ de las exportaciones en cada sector se mantienen en números muy bajos.

Tabla 3: $\mathrm{N}^{\circ}$ de empresas que concentran el $50 \%$ de las exportaciones

\begin{tabular}{|c|c|c|c|c|c|c|c|c|c|c|}
\hline Sector & 2000 & 2001 & 2002 & 2003 & 2004 & 2005 & 2006 & 2007 & 2008 & Promedio \\
\hline AGROEXPORTADORAS & 22 & 28 & 28 & 28 & 27 & 28 & 24 & 26 & 28 & 26 \\
\hline MADERA & 6 & 6 & 4 & 7 & 9 & 10 & 10 & 13 & 15 & 8 \\
\hline METAL-MECÁNICO & 10 & 8 & 12 & 20 & 16 & 11 & 14 & 14 & 14 & 13 \\
\hline PRENDAS DE VESTIR & 8 & 10 & 9 & 9 & 10 & 10 & 12 & 12 & 15 & 10 \\
\hline TEXTIL & 5 & 6 & 5 & 5 & 5 & 5 & 6 & 7 & 8 & 5 \\
\hline VARIOS & 8 & 8 & 8 & 7 & 6 & 6 & 6 & 6 & 7 & 6 \\
\hline MINERÍA NO METÁIICA & 8 & 6 & 6 & 5 & 5 & 5 & 4 & 4 & 5 & 5 \\
\hline MINERÍA TRADICIONAI & 4 & 4 & 4 & 4 & 3 & 3 & 4 & 4 & 5 & 3 \\
\hline $\begin{array}{l}\text { PESCA TRADICIONAL Y NO } \\
\text { TRAD. }\end{array}$ & 10 & 10 & 10 & 10 & 10 & 10 & 10 & 8 & 6 & 9 \\
\hline PETRÓLEO Y GAS NATURAL & 2 & 2 & 2 & 2 & 2 & 2 & 2 & 2 & 3 & 2 \\
\hline QUíMco & 13 & 12 & 13 & 15 & 15 & 16 & 14 & 13 & 13 & 13 \\
\hline $\begin{array}{l}\text { SIDERÚRGICOY } \\
\text { METALÚRGICO }\end{array}$ & 4 & 4 & 4 & 4 & 4 & 4 & 4 & 3 & 4 & 4 \\
\hline
\end{tabular}

\section{Principales productos}

El análisis de los principales productos del sector se realizó a nivel de 10 dígitos, para lo cual se estableció el siguiente procedimiento:

a) Se ordenó las partidas arancelarias con sus respectivos montos exportados de mayor a menor para cada periodo de la serie por sector.

b) Se halló la participación de cada partida arancelaria en cada periodo por sector y el valor acumulado de esta variable.

c) Se analizó e identificó la participación mínima requerida de las partidas arancelarias para explicar por lo menos el $40 \%$ de las exportaciones de cada periodo por sector (Por ejemplo: en el sector agroexportador existe una alta concentración de la exportación, de manera que en la serie analizada según el año pueden existir tres partidas o menos que explican el $45.39 \%$ o más de las exportaciones del sector. Para identificar los principales productos exportados se ha tomado aquellas partidas que al menos tengan el $5 \%$, debido a que las demás partidas no tienen un impacto significativo en el valor exportado del sector).

d) De acuerdo al resultado obtenido en el punto anterior, se colocó una restricción del $3 \%$ o $5 \%$ - según sea el caso- para hallar la cantidad de productos principales en cada periodo por sector.

Como resultado, la tabla 4 resume el número de partidas y su respectiva participación acumulada. Cabe resaltar que las partidas de cada periodo no concuerdan necesariamente con las de otros periodos, ya que solo se toman en cuenta aquellas que cumplan con la restricción propuesta en el punto (d) del procedimiento en su respectivo 
caso. Como se puede apreciar, el número de partidas promedio en todos los sectores no sobrepasa las 10 partidas.

Tabla 4: $\mathrm{N}^{\circ}$ de productos y su participación (por sector)

\begin{tabular}{|c|c|c|c|c|c|c|c|c|c|c|c|c|c|c|c|c|c|c|c|c|}
\hline \multirow{2}{*}{ Sector } & \multicolumn{2}{|c|}{2000} & \multicolumn{2}{|c|}{2001} & \multicolumn{2}{|c|}{2002} & \multicolumn{2}{|c|}{2003} & \multicolumn{2}{|c|}{2004} & \multicolumn{2}{|c|}{2005} & \multicolumn{2}{|c|}{2006} & \multicolumn{2}{|c|}{2007} & \multicolumn{2}{|c|}{2008} & \multicolumn{2}{|c|}{ Promedio } \\
\hline & $\mathbf{N}^{\circ}$ & $\%$ & $\mathbf{N}^{\circ}$ & $\%$ & $\mathbf{N}^{\circ}$ & $\%$ & $\mathbf{N}^{\circ}$ & $\%$ & $\mathbf{N}^{\circ}$ & $\%$ & $\mathbf{N}^{\circ}$ & $\%$ & $\mathbf{N}^{\circ}$ & $\%$ & $\mathbf{N}^{\circ}$ & $\%$ & $\mathbf{N}^{\circ}$ & $\%$ & $\mathbf{N}^{\circ}$ & $\%$ \\
\hline AGROEXPORTADORAS & 3 & $56 \%$ & 3 & $50 \%$ & 3 & $46 \%$ & 3 & $44 \%$ & 3 & $45 \%$ & 4 & $48 \%$ & 3 & $45 \%$ & 3 & $38 \%$ & 3 & $41 \%$ & 3 & $46 \%$ \\
\hline MADERA & 4 & $74 \%$ & 5 & $75 \%$ & 4 & $81 \%$ & 4 & $79 \%$ & 5 & $77 \%$ & 6 & $81 \%$ & 5 & $79 \%$ & 4 & $61 \%$ & 5 & $74 \%$ & 5 & $76 \%$ \\
\hline METAL-MECÁNICO & 7 & $38 \%$ & 6 & $46 \%$ & 6 & $27 \%$ & 5 & $18 \%$ & 6 & $23 \%$ & 5 & $32 \%$ & 5 & $25 \%$ & 4 & $23 \%$ & 4 & $21 \%$ & 5 & $28 \%$ \\
\hline PRENDAS DE VESTIR & 4 & $44 \%$ & 3 & $29 \%$ & 3 & $34 \%$ & 3 & $34 \%$ & 5 & $46 \%$ & 5 & $44 \%$ & 5 & $45 \%$ & 4 & $40 \%$ & 4 & $35 \%$ & 4 & $39 \%$ \\
\hline TEXTIL & 5 & $41 \%$ & 6 & $38 \%$ & 9 & $48 \%$ & 9 & $48 \%$ & 6 & $38 \%$ & 7 & $40 \%$ & 9 & $48 \%$ & 9 & $45 \%$ & 8 & $42 \%$ & 8 & $43 \%$ \\
\hline VARIOS & 2 & $38 \%$ & 2 & $42 \%$ & 4 & $54 \%$ & 4 & $56 \%$ & 3 & $49 \%$ & 3 & $48 \%$ & 4 & $49 \%$ & 3 & $38 \%$ & 4 & $39 \%$ & 3 & $46 \%$ \\
\hline MINERÍA NO METÁLICA & 6 & $44 \%$ & 7 & $55 \%$ & 7 & $59 \%$ & 6 & $61 \%$ & 4 & $57 \%$ & 4 & $57 \%$ & 5 & $67 \%$ & 5 & $65 \%$ & 4 & $56 \%$ & 5 & $58 \%$ \\
\hline MINERÍA TRADICIONAL & 4 & $80 \%$ & 5 & $81 \%$ & 4 & $84 \%$ & 4 & $85 \%$ & 5 & $86 \%$ & 5 & $85 \%$ & 5 & $88 \%$ & 5 & $86 \%$ & 5 & $86 \%$ & 5 & $85 \%$ \\
\hline $\begin{array}{l}\text { PESCA TRADICIONAL Y } \\
\text { NO TRAD. }\end{array}$ & 2 & $83 \%$ & 2 & $81 \%$ & 1 & $77 \%$ & 3 & $85 \%$ & 3 & $85 \%$ & 3 & $85 \%$ & 3 & $79 \%$ & 4 & $79 \%$ & 3 & $76 \%$ & 3 & $81 \%$ \\
\hline $\begin{array}{l}\text { PETRÓLEO Y GAS } \\
\text { NATURAL }\end{array}$ & 4 & $91 \%$ & 5 & $95 \%$ & 5 & $92 \%$ & 5 & $95 \%$ & 5 & $87 \%$ & 4 & $80 \%$ & 5 & $91 \%$ & 8 & $94 \%$ & 6 & $91 \%$ & 6 & $91 \%$ \\
\hline QÚ́MICO & 6 & $31 \%$ & 7 & $36 \%$ & 4 & $30 \%$ & 8 & $43 \%$ & 7 & $46 \%$ & 8 & $43 \%$ & 7 & $42 \%$ & 5 & $29 \%$ & 7 & $39 \%$ & 7 & $38 \%$ \\
\hline $\begin{array}{l}\text { SIDERÚRGICOY } \\
\text { METALÚRGICO }\end{array}$ & 5 & $65 \%$ & 5 & $67 \%$ & 4 & $52 \%$ & 7 & $64 \%$ & 6 & $61 \%$ & 3 & $46 \%$ & 3 & $53 \%$ & 5 & $62 \%$ & 5 & $64 \%$ & 5 & $59 \%$ \\
\hline
\end{tabular}

\section{Mercados destino}

En la tabla 5 se puede observar la evolución de los mercados de destino según sector. En la serie de tiempo se aprecia que los mercados de destino se han incrementado progresivamente en todos los sectores.

Tabla 5: Evolución de los mercados destino

\begin{tabular}{|l|c|c|c|c|c|c|c|c|c|}
\hline Sector & 2000 & 2001 & 2002 & 2003 & 2004 & 2005 & 2006 & 2007 & 2008 \\
\hline AGROEXPORTADORAS & 99 & 93 & 104 & 129 & 126 & 135 & 135 & 135 & 133 \\
\hline MADERA & 56 & 55 & 60 & 63 & 70 & 73 & 71 & 78 & 83 \\
\hline METAL-MECÁNICO & 73 & 66 & 73 & 90 & 89 & 92 & 97 & 109 & 100 \\
\hline PRENDAS DE VESTIR & 73 & 79 & 75 & 76 & 84 & 86 & 93 & 90 & 95 \\
\hline TEXTIL & 81 & 75 & 83 & 83 & 86 & 98 & 90 & 94 & 93 \\
\hline VARIOS & 81 & 81 & 89 & 89 & 88 & 102 & 108 & 113 & 118 \\
\hline MINERIA NO METÁLICA & 74 & 71 & 68 & 75 & 81 & 86 & 84 & 89 & 93 \\
\hline $\begin{array}{l}\text { MINERÍA TRADICIONAL } \\
\text { PESCA TRADICIONAL Y }\end{array}$ & 42 & 45 & 39 & 42 & 40 & 43 & 43 & 41 & 46 \\
\hline $\begin{array}{l}\text { NO TRAD. } \\
\text { PETROLEO Y GAS }\end{array}$ & 116 & 111 & 107 & 102 & 105 & 111 & 124 & 125 & 119 \\
\hline $\begin{array}{l}\text { NATURAL } \\
\text { QUIMMCO }\end{array}$ & 50 & 43 & 39 & 38 & 37 & 32 & 35 & 34 & 32 \\
\hline $\begin{array}{l}\text { SIDERURGICO Y } \\
\text { METALURGICO }\end{array}$ & 104 & 107 & 99 & 109 & 110 & 115 & 112 & 112 & 118 \\
\hline
\end{tabular}

Fuente: Aduanas - Peni. Elaboración propia.

Para hallar el número de mercados principales se siguió el mismo procedimiento que se utilizó para hallar el de productos principales.

a) Se ordenó las mercados con sus respectivos montos exportados de mayor a menor para cada periodo de la serie por sector.

b) Se halló la participación de mercado en cada periodo por sector y el valor acumulado de esta variable. 
c) Se analizó e identificó la participación mínima requerida de las partidas arancelarias para explicar por lo menos el $40 \%$ de las exportaciones de cada periodo por sector.

d) Según el resultado obtenido en el punto anterior, se colocó una restricción del $5 \%$ para hallar la cantidad de mercados principales en cada periodo por sector.

En la tabla 6 se resume la información obtenida del procedimiento aplicado. Cabe resaltar que para este caso se consideró la adición de las participaciones de los mismos mercados para cada periodo de la serie. Por eso, la tabla 6 solo indica el número de mercados una sola vez, a diferencia de la tabla de productos principales donde se utilizó la restricción para calcular el número de productos para cada periodo. Esto se realizó tomando en cuenta que los mercados son limitados, durables y poco volátiles a cambiar de nombre, por lo que no distorsiona los datos.

En la tabla 6 se puede observar que pocos mercados ocupan una alta concentración de las exportaciones, y un ejemplo distintivo es el caso de prendas de vestir, donde dos mercados concentran en promedio un $82 \%$ de las exportaciones del sector en el periodo analizado.

Tabla 6: № de mercados principales y su concentración (por sector)

\begin{tabular}{|c|c|c|c|c|c|c|c|c|c|c|c|}
\hline Sector & $\mathbf{N}^{\circ}$ & 2000 & 2001 & 2002 & 2003 & 2004 & 2005 & 2006 & 2007 & 2008 & Promedio \\
\hline AGROEXPORTADORAS & 4 & $62 \%$ & $62 \%$ & $62 \%$ & $60 \%$ & $61 \%$ & $60 \%$ & $60 \%$ & $60 \%$ & $56 \%$ & $60 \%$ \\
\hline MADERA & 3 & $83 \%$ & $82 \%$ & $84 \%$ & $82 \%$ & $82 \%$ & $86 \%$ & $87 \%$ & $84 \%$ & $84 \%$ & $84 \%$ \\
\hline METAL-MECÁNICO & 6 & $76 \%$ & $77 \%$ & $61 \%$ & $68 \%$ & $69 \%$ & $83 \%$ & $76 \%$ & $76 \%$ & $80 \%$ & $74 \%$ \\
\hline PRENDAS DE VESTIR & 2 & $78 \%$ & $78 \%$ & $80 \%$ & $83 \%$ & $85 \%$ & $85 \%$ & $84 \%$ & $84 \%$ & $82 \%$ & $82 \%$ \\
\hline TEXTIL & 7 & $53 \%$ & $56 \%$ & $53 \%$ & $56 \%$ & $59 \%$ & $58 \%$ & $61 \%$ & $64 \%$ & $70 \%$ & $59 \%$ \\
\hline VARIOS & 6 & $71 \%$ & $71 \%$ & $71 \%$ & $77 \%$ & $79 \%$ & $79 \%$ & $77 \%$ & $78 \%$ & $80 \%$ & $76 \%$ \\
\hline $\begin{array}{l}\text { MINERÍA NO } \\
\text { METÁLICA }\end{array}$ & 6 & $74 \%$ & $77 \%$ & $77 \%$ & $84 \%$ & $83 \%$ & $82 \%$ & $75 \%$ & $77 \%$ & $78 \%$ & $78 \%$ \\
\hline $\begin{array}{l}\text { MINERIA } \\
\text { TRADICIONAI }\end{array}$ & 5 & $58 \%$ & $46 \%$ & $50 \%$ & $51 \%$ & $51 \%$ & $62 \%$ & $59 \%$ & $61 \%$ & $63 \%$ & $56 \%$ \\
\hline $\begin{array}{l}\text { PESCA TRADICIONAL } \\
\text { Y NO TRAD. }\end{array}$ & 4 & $45 \%$ & $45 \%$ & $54 \%$ & $55 \%$ & $54 \%$ & $61 \%$ & $54 \%$ & $54 \%$ & $56 \%$ & $53 \%$ \\
\hline $\begin{array}{l}\text { PETRÓLEO Y GAS } \\
\text { NATURAL }\end{array}$ & 4 & $63 \%$ & $68 \%$ & $75 \%$ & $84 \%$ & $72 \%$ & $77 \%$ & $81 \%$ & $82 \%$ & $80 \%$ & $76 \%$ \\
\hline QUÍMICO & 7 & $66 \%$ & $70 \%$ & $67 \%$ & $68 \%$ & $73 \%$ & $70 \%$ & $63 \%$ & $65 \%$ & $71 \%$ & $68 \%$ \\
\hline $\begin{array}{l}\text { SIDERÚRGICOY } \\
\text { METALÚRGICO }\end{array}$ & 6 & $64 \%$ & $65 \%$ & $66 \%$ & $60 \%$ & $63 \%$ & $66 \%$ & $60 \%$ & $62 \%$ & $73 \%$ & $64 \%$ \\
\hline
\end{tabular}

Fuente: Aduanas - Pení. Elaboración propia.

\section{Continuidad de las empresas exportadoras}

Las empresas exportadoras se dividen en dos grupos: las exportadoras permanentes, que se definen como aquellas que se mantienen en la actividad exportadora todos los años en el periodo de estudio; y las exportadoras esporádicas, aquellas que una vez que han logrado entrar a los mercados internacionales, deciden salir en algún año posterior (Álvarez, 2004).

Para el presente documento se consideraron como empresas exportadoras permanentes aquellas que hayan exportado por lo menos tres años consecutivos desde el momento que inician su primera exportación dentro del periodo 2000-2008. 
En la tabla 7 se refleja la cantidad de empresas clasificadas como esporádicas y su representación ante el total de empresas del sector. Como se puede ver, existe una alta tasa de empresas esporádicas en todos los sectores.

Tabla 7: N de empresas esporádicas y su representación del total de empresas (por sector)

\begin{tabular}{l|c|c|}
\hline Sector & No & $\%$ \\
\hline AGROEXPORTADORAS & 3643 & $84.15 \%$ \\
\hline MADERA & 2711 & $92.05 \%$ \\
\hline METAL-MECÁNICO & 6323 & $94.08 \%$ \\
\hline PRENDAS DE VESTIR & 4042 & $84.95 \%$ \\
\hline TEXTIL & 2739 & $89.83 \%$ \\
\hline VARIOS & 6539 & $88.77 \%$ \\
\hline MINERÍA NO METÁLICA & 3026 & $72.34 \%$ \\
\hline MINERÍA TRADICIONAL & 319 & $81.54 \%$ \\
\hline PESCA TRADICIONAL Y & 1272 & \\
\hline NO TRAD. & 200 & $84.40 \%$ \\
\hline PETRÓLEO Y GAS & 3796 & $92.13 \%$ \\
\hline NATURAL & & $89.27 \%$ \\
\hline QUÍMICO & 1563 & $91.99 \%$ \\
\hline SIDERURGICO Y & & \\
METALÚRGICO & &
\end{tabular}

Fuente: Aduanas - Perí. Elaboración propia.

El tabla 8 muestra el número de empresas que realizaron actividades de exportación solamente por un año, dentro de la serie 2000 al 2007. La data de las empresas que exportaron solo el 2008 no se toma en cuenta dado que, para identificar si exportaron únicamente este año y no los posteriores, se requeriría información del 2009 en adelante, y el objetivo del presente trabajo es analizar la continuidad para el periodo 2000-2008. Debido a que el número de empresas que exportan solamente por un año son distintas entre los periodos, es posible calcular el total de empresas que solo exportaron una vez, como se muestra en la última columna de la tabla.

Tabla 8: № de empresas que exportan solo por un año (2000-2007)

\begin{tabular}{|c|c|c|c|c|c|c|c|c|c|}
\hline Sector & 2000 & 2001 & 2002 & 2003 & 2004 & 2005 & 2006 & 2007 & Tota \\
\hline AGROEXPORTADORAS & 307 & 146 & 150 & 163 & 194 & 220 & 232 & 213 & 1625 \\
\hline MADERA & 187 & 124 & 173 & 168 & 184 & 198 & 195 & 211 & 1440 \\
\hline METAL-MECÁNICO & 414 & 389 & 413 & 440 & 425 & 483 & 479 & 537 & 3580 \\
\hline PRENDAS DE VESTIR & 188 & 154 & 169 & 184 & 188 & 255 & 268 & 284 & 1690 \\
\hline TEXTIL & 156 & 111 & 141 & 146 & 186 & 205 & 210 & 219 & 1374 \\
\hline VARIOS & 392 & 308 & 292 & 362 & 382 & 402 & 480 & 502 & 3120 \\
\hline MINERÍA NO METÁLICA & 14 & 5 & 7 & 20 & 13 & 17 & 15 & 15 & 106 \\
\hline MINERIA TRADICIONAL & 29 & 17 & 15 & 15 & 16 & 13 & 31 & 17 & 153 \\
\hline $\begin{array}{l}\text { PESCA TRADICIONAL Y } \\
\text { NO TRAD. }\end{array}$ & 113 & 59 & 54 & 61 & 63 & 59 & 70 & 64 & 543 \\
\hline $\begin{array}{l}\text { PETROLEO Y GAS } \\
\text { NATURAL }\end{array}$ & 7 & 7 & 11 & 19 & 11 & 16 & 20 & 16 & 107 \\
\hline QUÍMICO & 209 & 178 & 194 & 214 & 214 & 258 & 293 & 308 & 1868 \\
\hline $\begin{array}{l}\text { SIDERURGICOY Y } \\
\text { METALÚRGICO }\end{array}$ & 93 & 85 & 96 & 97 & 74 & 115 & 122 & 139 & 821 \\
\hline
\end{tabular}

Fuente: Aduanas - Perí. Elaboración propia.

En la tabla 9 se puede observar todos los resultados (OR) que se obtuvieron después de aplicar el procedimiento estadístico mencionado. EI OR para el primer modelo -NA del sector agroexportador es de 1.507, e indica que por cada año adicional que la empresa exporte, la probabilidad de permanencia en el mercado se incrementa en un $50.7 \%$ (1.507-1 x 100\%). El OR del segundo modelo (RACHA) es de 3.066 e indica 
que por cada año que se agregue a la continuidad de la empresa, la probabilidad de que permanezca en el mercado internacional se incrementa en un $206.6 \%$. El OR del tercer modelo (CON) es de 1.882, e indica que por cada año adicional a la continuidad de la empresa, la probabilidad de que permanezca en el mercado se incrementa en un $88.2 \%$. Asimismo, en el primer modelo se deduce que el valor estimado del logaritmo de la razón de probabilidades toma valores positivos cuando el valor de NA>4.43. Esto indica que la probabilidad que una empresa se mantenga de manera exitosa en su actividad exportadora será mayor de 0.5 , y la razón de probabilidades será mayor de 1 cuando la empresa tenga cinco años o más en la actividad exportadora.

Tabla 9: Resultados OR obtenidos

\begin{tabular}{|c|c|c|c|c|c|}
\hline \multirow{2}{*}{\multicolumn{2}{|c|}{ Sector }} & \multicolumn{2}{|c|}{ Pequeñas empresas } & \multicolumn{2}{|c|}{ Grandes empresas } \\
\hline & & Variables & OR & Variables & or \\
\hline \multirow{4}{*}{1} & \multirow{4}{*}{ Agropectunio y agroindustrias } & NA & 1.507 & $\mathrm{NA}$ & 1.378 \\
\hline & & Racha & 3.066 & Rachn & 1.9 \\
\hline & & Con & 1.882 & Con & 1.775 \\
\hline & & $-\mathrm{NA}>$ & 4.43 & NA> & 2.809 \\
\hline \multirow{4}{*}{2} & \multirow{4}{*}{ Madera } & NA & 1.467 & NA & 1.456 \\
\hline & & Racha & 2.552 & Racha & 2.142 \\
\hline & & Con & 1.527 & Con & 1.684 \\
\hline & & -NA> & 4.88 & NA> & 3.85 \\
\hline \multirow{4}{*}{3} & \multirow{4}{*}{ Metal-Mecinico } & $\mathrm{NA}$ & 1.625 & NA & 1.656 \\
\hline & & Rachn & 3.025 & Rachs & 1.945 \\
\hline & & Con & 1.565 & Con & 1.904 \\
\hline & & -NA> & 3.94 & NA $>$ & 3.51 \\
\hline \multirow{4}{*}{4} & \multirow{4}{*}{ Mineria no metíliea } & $\mathrm{NA}$ & 1.41 & $\mathrm{NA}$ & 1.279 \\
\hline & & Racha & 2.217 & Racha & 1.606 \\
\hline & & Con & 1.869 & Con & 1.527 \\
\hline & & -NA> & 4.67 & NA> & 1.65 \\
\hline \multirow{4}{*}{5} & \multirow{4}{*}{ Mineris tradicional } & NA & 1.929 & NA & 1.517 \\
\hline & & Racha & 4.581 & Racha & 2.173 \\
\hline & & Con & 1.718 & Con & 1.971 \\
\hline & & -NA> & 4.1 & NA $>$ & 3.27 \\
\hline \multirow{4}{*}{6} & \multirow{4}{*}{ Perca } & $\mathrm{NA}$ & - & NA & 1.41 \\
\hline & & Rachn & - & Racha & 1.78 \\
\hline & & Con & - & Con & 1.59 \\
\hline & & -NA> & - & NA> & 5.31 \\
\hline \multirow{4}{*}{7} & \multirow{4}{*}{ Peres no tadicions] } & NA & 1.464 & $\mathrm{NA}$ & 1.27 \\
\hline & & Racha & 2.141 & Racha & 1.742 \\
\hline & & Con & 1.807 & Con & 1.735 \\
\hline & & -NA> & 5.92 & NA> & 3.03 \\
\hline \multirow{4}{*}{8} & \multirow{4}{*}{ Petróleo y gas watural } & NA & 1.241 & $\mathrm{NA}$ & 2.057 \\
\hline & & Rachn & 4.3 & Rachn & 3.933 \\
\hline & & Con & 1.419 & Con & 3.518 \\
\hline & & -NA> & 4.34 & NA> & 1.57 \\
\hline \multirow{4}{*}{9} & \multirow{4}{*}{ Prendas de vestir } & $\mathrm{NA}$ & 1.353 & $\mathrm{NA}$ & 1.277 \\
\hline & & Racha & 2.3 & Rachn & 1.821 \\
\hline & & Con & 1.572 & Con & 1.724 \\
\hline & & -NA> & 4.31 & NA> & 1.15 \\
\hline \multirow[t]{3}{*}{10} & Quimico & $\begin{array}{l}\text { NA } \\
\text { Racha }\end{array}$ & $\begin{array}{l}1.587 \\
2.654\end{array}$ & $\begin{array}{l}\text { NA } \\
\text { Rachn }\end{array}$ & $\begin{array}{l}1.536 \\
1.986\end{array}$ \\
\hline & & Con & 1.694 & Con & 1.951 \\
\hline & & -NA> & 3.75 & NA> & 2.99 \\
\hline & & $\mathrm{NA}$ & 1.528 & NA & 1.482 \\
\hline 11 & Siderírgico & Racha & 3.09 & Rachn & 2.091 \\
\hline 11 & suderurguso & Con & 1.544 & Con & 1.883 \\
\hline & & -NA> & 3.79 & NA> & 3.26 \\
\hline & & $\mathrm{NA}$ & 1.448 & NA & 1.554 \\
\hline 12 & Terti & Racha & 2.253 & Rachn & 2.193 \\
\hline 12 & Textal & Con & 1.648 & Con & 2.647 \\
\hline & & -NA> & 4.07 & $+\mathrm{NA}>$ & 2.46 \\
\hline
\end{tabular}


Asimismo, la tabla 9 muestra valores representativos y coherentes para todos los sectores, a excepción del sector pesca en empresas pequeñas, debido a que las estimaciones del modelo se muestran poco confiables e inconsistentes.

\section{Conclusiones}

Después del análisis extensivo, tanto descriptivo como estadístico, se puede concluir que el Perú registró una excelente tasa de crecimiento para sus exportaciones, de manera que el promedio de crecimiento anual de los sectores exportadores fue de $19.2 \%$. Asimismo, las exportaciones del Perú se encuentran altamente concentradas, pues la cantidad de empresas que agrupan el $50 \%$ o más de las exportaciones de cada sector fluctúa entre 2 y 13 empresas, siendo la excepción el sector agroexportador, representado por 26 empresas. Por otro lado, a nivel de productos representados por partidas arancelarias también refleja una alta concentración donde, en promedio, cinco partidas arancelarias concentran el 56\% de las exportaciones del Perú. De igual manera, a nivel de mercados también se da una alta concentración, ya que los sectores tienen entre 2 y 7 mercados que concentran valores superiores al $50 \%$ de las exportaciones.

Respecto a las empresas exportadoras se encontró que en promedio el $87 \%$ son esporádicas. Este porcentaje llega hasta el $94.08 \%$ en el caso del sector de metalmecánico, lo que revela la falta de continuidad y compromiso con los mercados internacionales de las empresas exportadoras del Perú. Esto significa una oportunidad para poder definir políticas que contribuyan a darles mayor continuidad.

La alta concentración de las exportaciones del Perú a nivel de empresas, productos y mercados, así como el contar con un alto porcentaje de empresas exportadoras esporádicas, revelan la necesidad de identificar y analizar cuáles son las barreras que impiden a la empresa exportadora mejorar su desempeño. Esto implica que se debe profundizar la investigación en este campo, a efecto de tener un conocimiento más cabal sobre las necesidades de los empresarios para mantenerse en los mercados internacionales., según Gray (1997), es necesario que el estudio identifique tanto factores externos como internos de la empresa (Hurtado Torres, 2002) (Morgan, 1997) (Leonidou, Katsikeas, \& Piercy, 1998).

En cuanto a los resultados del análisis estadístico, estos se presentan de acuerdo a cada variable del modelo tanto para la pequeña como para la gran empresa:

a. En el caso de las empresas pequeñas, los modelos indican que por cada año adicional (NA) de permanencia en el mercado exportador, su probabilidad de éxito se incrementa en promedio en un $40 \%$. En cuanto a las empresas grandes, los modelos indican que por cada año adicional de permanencia en el mercado exportador, su probabilidad de éxito se incrementa en promedio en un 35\%.

b. Para las empresas pequeñas, los modelos indican que por cada año adicional continuo (Racha) en el mercado exportador, su probabilidad de éxito se incrementa, en promedio, en más de $100 \%$. Para las empresas grandes, est probabilidad se incrementa, en promedio, en más de $80 \%$.

c. Igualmente, por cada año adicional continuo de actividad exportadora antes del 2008 (CON), para las empresas pequeñas la probabilidad de permanencia en el 
mercado se incrementa en promedio en más de 50\%; y en las empresas grandes, en más de $60 \%$.

d. Además, para que una empresa se consolide dentro del sector exportador se requiere que su experiencia supere los cuatro años para empresas pequeñas y tres años para las empresas grandes.

e. Los modelos estimados muestran altos niveles de predicción de las probabilidades de que las empresas permanezcan en el mercado y, debido a que las variables incluidas en los modelos están relacionadas con la continuidad de las empresas en su actividad exportadora, se puede concluir que los resultados confirman la hipótesis presentada, donde se afirma que la continuidad en la actividad exportadora de una empresa es fundamental para su éxito futuro. Esto también confirma lo mencionado por Alonso y Donoso (2000), quienes sostienen que la acumulación de experiencia en los mercados exteriores reduce los costos generados en dichos mercados y genera economías de aprendizaje, además de incrementar las probabilidades de tener mejores resultados que estimulen la exportación.

f. Para el caso de las empresas exportadoras grandes, los modelos estimados demuestran que la continuidad en la actividad exportadora es un factor determinante en el pronóstico de la probabilidad de éxito.

g. Los modelos nos brindan una explicación significativa en la estimación de la probabilidad de permanencia de una empresa exportadora, pues nos otorgan una relación de probabilidades. Esto significa que por cada año adicional en que permanece la empresa exportando, su probabilidad de permanencia en el mercado se incrementa en relación a la probabilidad de que deje el mercado de exportaciones.

\section{Referencias bibliográficas}

Alonso, José Antonio \& Donoso, Vicente (2000). Modelización del comportamiento de la empresa exportadora española. ICES Sector Exterior Español (788). España

Álvarez, Roberto (2004). Desempeño exportador de las empresas chilenas: algunos hechos estilizados. CEPAL, 121-134. Chile

Arteaga, Jesús \& Medina, Diego (2006). La importancia del tamaño en la actividad exportadora: Una evaluación del caso de las pymes españolas exportadoras no consolidadas. Boletín Económico del ICE (2883). España.

Baez, John \& Lauda, Aaron (s.f.). Deep Beauty: Mathematical Innovation and the Search for an Underlying Intelligibility of the Quantum World. EE.UU.

Barreiro Fernández, José; Losada Pérez, Fernando; Ruzo Sanmartín, Emilio; Navarro García, Antonio (2004). Orientación al mercado y resultado exportador: análisis de la empresa exportadora gallega a través de un modelo logit. España

Bazán, José Luis \& Millones, Oscar (2008). Una clasificación de modelos de regresión binaria asimétrica: el uso de BAYES - PUCP en una aplicación sobre la decisión del cultio ilícito de hoja de coca. Economía, 17-32. Perú 
Bernard, Andrew \& Jensen, Bradford (1999). Exceptional Exporter Performance: Cause, Effect or Both? Journal of International Economics, 1-25. EE.UU.

Bettis, Richard \& Prahalad, Coimbatore Krishnarao (1995). The Dominant Logic: Retrospective and Extension. Strategic Management Journal, 16, 5-14. EE.UU.

Bonaccorsi, Andrea (1992). On the relationship between firm size and export intensity. Journal of International business studies, 605-635. Ottawa, Canadá

Calderón García, Haydeé \& Fayos Gardó, Teresa (2004). Análisis de la relación entre el compromiso exportador y las ayudas a la internacionalizacion de las empresas. Investigaciones Europeas de Dirección y Economía de la Empresa, 10(2), 201-220. Valencia, España

Fernández, Horacio \& Pérez, Fredy (2005). El modelo logístico: Una herramienta estadística para evaluar el riesgo de crédito. Ingenierías, 55-75.

Cavusgil, Tamer \& Nevin, John (1981). Internal Determinants of Export Marketing Behavior: An Empirical Investigation. Journal of Marketing Research, 114-119. EE.UU.

Cavusgil, Tamer \& Zou, Shaoming (1994). Marketing Strategy-Performance Relationship: An Investigation of the Empirical Link in Export Market Ventures. The Journal of Marketing, 58 (1), 1-21. EE.UU.

Cyert, Richard \& March, James (1992). A Behavioral Theory of the Firm. New Jersey: Prentice-Hall Inc.

De Lucio, Juan José; Mínguez, Raúl; Valero, Manuel \& Mednik, Gregorio (2008). -Permanencia de las empresas en la exportación: Una mirada a las características de su actividad exteriorll. En: Tribuna de economía (840), 179-195. España

Del Río, María Luisa \& Varela, María Concepción (2006). Características de los directivos y y rendimiento exportador en las pymes españolas. ESIC Market, 243-254.

Grant, Robert (2004). Dirección estratégica: conceptos, técnicas y aplicaciones. Madrid: Thomson - Civitas.

Gray, Brendan (1997). Profiling Managers to Improve Export Promotion Targeting. Journal of International Business Studies, 387-420.

Gujarati, Damodar (1999). —Econometríall. En: D. Gujarati, Econometría (págs. 529564).

Huerta, E., \& Labeaga, J. M. (1992). Análisis de la decisión de exportar: una aproximación con datos microeconómicos. Investigaciones Económicas, 41-47.

Hurtado Torres, Nuria Esther (2002). La PYME y las estrategias de Internacionalización. Granada: Universidad de Granada.

Jiménez, Francisco \& Espinoza, Carlos (2007). Costos Industriales. Cartago: Editorial Tecnológica de Costa Rica. Costa Rica 
Johanson, Jan \& Vahlne, Jan-Erik (1990). -The Mechanism Of Internationalisationll. En: International Marketing Review, Vol, 7, Iss. 4, pp.11 - 24.

Johanson, Jan \& Vahlne, Jan-Erik (1977). The internationalization process of the firm A Model of Knowledge Development and increasing Foreign Market Commitments. Journal International Business Studies , 23 - 32.

Johanson, Jan \& Vahlne, Jan-Erik (2009). The Uppsala internationalization process model revisited: From liability of foreignness to liability of outsidership. Journal of International Business Studies, 1411-1431.

Johanson, Jan \& Wiedersheim, Paul (1975). The internationalization of the firm - four swedish cases. The Journal of Management Studies.

Katsikeas, Constantine \& Piercy, Nigel (1996). Determinants of export performance in a European context. European Journal of Marketing, 6-35. EE.UU.

Kogut, Bruce \& Zander, Udo (2003). Knowlegde of the firm and the evolutionary theory of the multinacional corporation. Journal of International Bussines Studies, 625-645.

Leonidou, Leonidas; Katsikeas, Constantine \& Piercy, Nigel (1998). Identifying managerial influences on exporting: Past research and future directions. Journal of International Marketing, 74-102.

Madrid, Antonia \& Garcia, Domingo (2004). Influencia del tamano, la antiguedad y el rendimiento sobre la intensidad exportadora de la PyMe industrial espanola. Sector Exterior Espanol (817). Espana.

Madsen, Tage Koed (1998). \Executive insights: managerial judgment of export performance.. En: Journal of International Marketing, Vol. 6, no 3, pp. 82-93.

Martin Armario, Julia; Rastrollo Horrillo, M. Angeles; Gonzalez Robles, Eva M. (2009). La internacionalizacion de la empresa: el conocimiento experimental como derterminante del resultado en mercados exteriores. Cuadernos de Economia y Direccion de la Empresa (39), 123-150. Espana

Morgan, Robert (1997). Export Stimuli and Export Barriers: evidence from empirical research studies. European Business Review, 97, 68-79.

Navarro, Antonio; Acedo, Francisco; Robson, Matthew; Ruzo, Emilio \& Losada, Fernando (2010). \Antecedents and Consequences of Firms f Export Commitment: An Empirical Study.. En: Journal of International Marketing , 18 (3), 41-61.

Nguyen, Hong Thi-Tuyet (2000). The Determinants of export marketing activities of small and medium-sized manufacturing firms in Germany. Bell \& Howell Information and Learning Company. Alemania

Peng, Mike (2009). Global Business. Dallas: South-Western Cengage Learning. EE.UU.

Pla, Barber \& Leon, Fidel (2004). Direccion de Empresas Internacionales. Madrid: Pearson Prentice Hall. EspaNa 
Prahalad, Coimbatore Krishnarao \& Hamel, Gary (1990). IThe Core Competence of the Corporation.. En: Harvard Business Review, On Point , 1- 15. EE.UU.

Raymond, Mary Anne; Kim, Jonghoon \& Shao, Alan T. (2001). Export Strategy and Performance: A comparison of exporters in a developed market and an emerging market. Journal of Global Marketing, 15, 5-25.

Rialp, Alex (1999). Los Enfoques Micro-organizativos de la Internacionalizacion de la Empresa: Una Revision y Sintesis de la Literatura. Informacion Comercial Espanola (ICE), Octubre 1999, no 781, 117-128.

Roberts, Mark \& Tybout, James (1997). La decision de exportar en Colombia: un modelo empirico de entrada con costos hundidos. ESPE , 61-100. Colombia

Roots, Franklin (1994). Entry Strategies for International Markets. Nueva York: Lexington Books.

Suárez, Sonia (2004). -Determinantes de la actividad exportadora en el sector vitivinícola españolll. En: Vector plus: miscelánea científico - cultural , 79 - 87. España

Suárez, Sonia; Olivares, Arístides \& Galván, Inmaculada (2002). La expansión de los mercados de exportación y el tamaño empresarial: el caso de las empresas exportadoras canarias. Sector Exterior Español ICE (802). España.

Welch, Lawrence S. \& Luostarinen, Reijo (1988). Internationalisation: Evolution of a Concept. Journal of General Management, Vol. 14 (2), 34-55. 